\title{
Make the Adder Count: population trends from a citizen science survey of UK adders
}

\author{
Emma Gardner ${ }^{1}$, Angela Julian², Chris Monk ${ }^{3}$ \& John Baker ${ }^{4}$ \\ ${ }^{1}$ School of Biological Sciences, University of Reading, Whiteknights, Reading, RG6 6AS, UK \\ ${ }^{2}$ Amphibian and Reptile Groups of UK (ARG UK), 82 Gidley Way, Horspath, Oxford, OX33 1TG, UK \\ ${ }^{3}$ Amphibian and Reptile Groups of UK (ARG UK), Hilburn, Chapel Lane, Middleton, Matlock, Derbyshire, DE4 4NF, UK \\ ${ }^{4}$ Amphibian and Reptile Groups of UK (ARG UK), 34 Barons Court, Halesworth, Suffolk, IP19 8EJ, UK
}

Concern has been growing about the status of UK adder populations, with expert opinion reporting widespread declines. Assessing the true scale of these declines, however, has been hampered by a lack of quantitative data. Make the Adder Count began in 2005 as a national surveillance programme collecting standardised counts of adders lying-out after emerging from hibernation. 260 sites have contributed data, confirming a significant decline, on average, across sites with small populations, while the few with large populations ( $<10 \%$ of sites) are weakly increasing. If these trends continue, within 15-20 years, adders will be restricted to a few large population sites, significantly increasing the extinction risk for this priority species in the UK. Public pressure/disturbance was reported as the most frequent negative factor affecting sites, followed by habitat management and habitat fragmentation. Negative impacts from habitat management were reported almost as frequently as positive impacts, suggesting many management plans do not adequately consider the requirements of adders. The dataset also demonstrated earlier emergence among males, in warmer springs and at more northerly sites.

Key words: adders, population trends, habitat management, conservation, emergence survey, citizen science

\section{INTRODUCTION}

$\mathrm{T}_{\mathrm{B}}^{\mathrm{h}}$ diter he adder, Vipera berus, was added to the UK Biodiversity Action Plan list of priority species in 2007. Although historically widespread (Taylor, 1963; Arnold, 1995), changes in status derived from biological recording data indicate adder numbers in the UK have been in decline since the 1930s (Cooke \& Arnold, 1982). A questionnaire carried out by Cooke \& Scorgie (1983) reported declines in three out of 12 Nature Conservancy Council regions, rising to six out of 12 regions in a follow-up assessment (Hilton-Brown \& Oldham, 1991). Baker et al. (2004) evaluated population trends at specific sites, again via a questionnaire, and showed that more populations were judged to be decreasing than increasing, with population decreases particularly prevalent in the Midlands. A study combining historical records with adder habitat requirements and land cover data suggested the species had suffered a $39 \%$ range reduction by 2011 (Gleed-Owen \& Langham, 2012).

The adder shares the same degree of legal protection as other widespread reptile species in the UK (e.g. grass snake, Natrix helvetica), however, aspects of its ecology mean that it may be less able to survive in an anthropogenic landscape and hence may deserve greater conservation prioritisation. The adder is confined to specific habitats, being found mostly in woodland, heathland and moorland (Swan \& Oldham, 1993; Arnold, 1995). As a result, it demonstrates a patchy distribution over much of its range (Viitanen, 1967; Prestt, 1971; Madsen \& Shine, 1992). Even within areas of suitable habitat, it is usually confined to habitat features or patches of warm micro-habitat, such that Gleed-Owen and Langham (2012) suggest adders may occupy as little as $29 \%$ of the potentially suitable habitat in England, based on the species' habitat preferences. Adders are also relatively sedentary (e.g. compared with the grass snake), with small home ranges (Langton \& Beckett, 1995), so that populations confined to specific localities can be effectively isolated. Moreover, many populations are small (fewer than 10 adults; Baker et al., 2004) and the combination of these factors means that adders are prone to inbreeding depression (Madsen et al., 1996) and local extinctions, with limited chances of recovery.

Despite growing concern for the status of UK adder populations and their high vulnerability to negative impacts from increases in human activity and land-use change, no co-ordinated programme for collecting data on adder numbers existed in the UK, prior to 2005. All previous assessments of adder status have been based on either expert opinion (Cooke \& Scorgie, 1983; Hilton- 
Brown \& Oldham, 1991; Baker et al., 2004) or analysis of ad-hoc records (Cooke \& Arnold, 1982; Gleed-Owen \& Langham, 2012). Recent initiatives such as Add an Adder (run by Amphibian and Reptile Conservation; ARC) and Record Pool (Amphibian and Reptile Groups of the UK and ARC) similarly collect ad-hoc sightings, while the only systematic survey of UK amphibians and reptiles - the National Amphibian and Reptile Recording Scheme - has yielded very little data on adders, with adders recorded in only $7 \%$ ( 22 squares) of the $3101 \mathrm{~km}$ squares surveyed between 2007 - 2012 (Wilkinson \& Arnell, 2013).

Assessing the true scale of adder declines has therefore been hampered by a lack of long-term, quantitative data. Make the Adder Count (MTAC) was set up in 2005 to change this, by providing the first longterm, national surveillance programme designed to collect standardised data on adder numbers across the UK. Adders hibernate over winter, regularly using the same hibernacula year after year and often hibernating communally (e.g. Prestt, 1971; Phelps, 2004), which concentrates populations in specific locations. After emerging from hibernation in spring, adult snakes (particularly males) bask for prolonged periods close to the hibernaculum (Prestt, 1971; Phelps, 2004), during which time they are detected relatively easily by surveyors. Experienced volunteer surveyors (those already involved in reptile fieldwork, rather than recruited from a wider public appeal) were encouraged to register known hibernacula with MTAC and submit spring counts of basking adders.

The aims of MTAC were threefold:

1. To test the potential of repeated spring counts by volunteer surveyors as a viable method for collecting quantitative data on adder populations.

2. To derive population trends for UK adders and assess the current factors affecting adder populations.

3. To locate hibernation sites to inform habitat management.

In this paper, we present results from the first 11 years of MTAC, which confirm, for the first time with standardised quantitative data, significant declines across the majority of adder sites. We also identify three key factors which must be addressed, if the adder is to have a viable future in the UK.

\section{Methods}

MTAC is a citizen science project that specifically targets experienced surveyors with knowledge of adder overwintering sites. Potential participants were contacted through a national network of local volunteer groups (the Amphibian and Reptile Groups of the UK) and an annual national conference (the Herpetofauna Workers Meeting). Many of the participants already monitored local adder populations, so MTAC aimed to standardise and collate count data that participants may have already been recording.

\subsection{Survey Methodology}

Sites were defined as a single hibernation site or aggregation area or, where hibernation sites were dispersed rather than aggregated (such that adders basked individually rather than communally), a repeatable survey transect. Surveyors made multiple visits to their sites between February and May. MarchApril was recommended as the optimum time window, but surveyors were asked to judge the date of site visits and appropriate weather conditions according to their own experience of the site, in recognition of geographic variation. A minimum of three visits was required, with five or six visits recommended. On each visit, surveyors recorded the number of adult snakes observed basking after emergence from hibernation (i.e. excluding juveniles, which were identified by their smaller body size and are rarely encountered during the chosen survey period). Although it is possible to identify individual adders via head markings (Sheldon \& Bradley, 1989), thus enabling population size estimates via mark-recapture techniques, we considered collecting the necessary data too labour intensive for most volunteer surveyors and therefore limited data collection to recording number of adults observed (regardless of whether they have been previously encountered or not), in order to maximise participation. If it was possible to visually sex the adders without disturbing them, and if confident to do so, the surveyor was asked to record male and female adders separately (sexual dichromatism in adders is normally sufficient to distinguish between the sexes in adults; Beebee \& Griffiths, 2000).

Surveyors were also asked to provide information about the site itself, including site area (i.e. the area of suitable adder habitat around the surveyed hibernation site/aggregation/transect on a two-point scale: $0-5$ ha and more than 5 ha), connectivity (how well connected the surveyor considered the site was to other sites on a four-point scale: 1 = completely isolated by many kilometres, 2 = isolated from nearby sites by sub-optimal habitat, 3 = linked by corridors, 4 = part of a larger group of populations occupying more or less continuous habitat) and factors judged to affect the population. For the latter, 12 categories were given and participants could select any of these as having either a negative or positive effect. The factors were: public pressure through disturbance, habitat management, habitat fragmentation/isolation, neglect/succession, persecution, fire, predation, forestry operations, building development, agricultural changes, introduction (conservation) and introduction (development mitigation). An option to record factors that did not fall into these categories was also provided. The categories were based on those used in previous questionnaire studies (Cooke \& Scorgie, 1983; HiltonBrown \& Oldham, 1991; Baker et al., 2004) to facilitate comparison.

\subsection{Data Analysis}

Data analysis was carried out using $R$ version 3.4.3 ( $R$ Core Team 2017), with maps and nearest-neighbouringsite analysis generated using QGIS version 2.10.1 (QGIS Development Team 2015).

\subsubsection{Population Trends}

For each site $j$ in each year $i$ we used the peak count $k_{j, i}$ as an index of population size, where the peak count was 
defined as the maximum number of adders recorded at site $j$ on any one visit during year $i$. Throughout the survey season, adders are emerging, basking near the hibernaculum and then dispersing into the surrounding habitat, with the timing of emergence and length of this basking period not only subject to individual variation, but also affected by the individual's sex and breeding status (both of which are often unknown). This variation in detectability between individuals and for the same individual over time prevents the use of more complex statistical models which use repeat visits to disentangle detectability and abundance assuming constant detectability and closed populations (e.g. N-mixture models; Royle, 2004; Ward et al., 2017; Barker et al., 2018). The peak count therefore remains the most robust index of population size available under these conditions with the available data.

For each site, we calculated the mean peak count $\left(K_{j}\right)$ across its entire time series and used this to split the sites into two groups: those with $K_{j} \leq K_{\text {threshold }}$, which we assume correspond to small population sites, and those with $K_{j}>K_{\text {threshold }}$, which we assume correspond to large population sites (see next section for determination of $K_{\text {threshold }}$. We analysed the population trends for these two groups separately.

Only sites with three or more years of data were included in the population trend analysis. Ideally, overall population trends would be modelled using site-level peak count data in a generalised linear model with a fixed site effect. However, such models only produced adequate fits for the large populations sites, which have relatively well-sampled and well-behaved time series, which are roughly consistent with linear trends. For the small population sites, linear models provided a poor fit to much of the site-level data, due in part to the nature of their population trends. Adopting a more complicated functional form for the time dependence (e.g. allowing for linear trends in some sites and exponential declines in others) was not feasible, given that many sites had only a few years of data and so had insufficient data to constrain multiple parameters. Consequently, we used the method described below, which accounts for variation in survey effort and determines comparable average population trends for the two groups, without imposing any functional forms at site level:

1. For each site, we calculated its normalised peak count per year as:

$$
k_{n o r m, j, i}=\frac{k_{j, i}}{K_{j}}
$$

This preserves the fractional change in population size.

2. For each year, we calculated the weighted mean normalised peak count (i.e. mean fractional change in population size) across all sites surveyed that year as:

$$
K_{n o r m, i}=\frac{\sum_{j} w_{j, i} k_{n o r m, j, i}}{\sum_{j} w_{j, i}}
$$

where $w_{i, i}$ is an individual weighting factor attributed to the normalised peak count from each of the sites surveyed in that year. The larger the number of visits that have gone into determining a peak count, the more confident we are that that peak count is representative of the population size at that site in that year. We therefore based the weighting factor for each peak count on the number of site visits $\left(n_{\text {visits }}\right)$ used to determine that peak count. The weights were set as follows: $w=1$ for $n_{\text {visits }}<3$, $w=2$ for $3 \leq n_{\text {visits }}<6$ and $w=3$ for $n_{\text {visits }} \geq 6$, i.e. for three sites with normalised peak counts 1.2, 2.4 and 3.5, derived from 4,2 and 6 visits, respectively, we would calculate a mean normalised peak count of $((2 \times 1.2)+(1 \times 2.4)+(3$ $\times 3.5)) /(2+1+3)=2.55$.

3. We calculated the uncertainty (standard error) on each mean normalised peak count as:

$$
\alpha_{K_{n o r m, i}}=\frac{\sigma_{k_{n o r m, j, i}}}{\sqrt{N_{i}}}
$$

where the standard deviation of the normalised peak counts, $\sigma_{k_{n o r m, j, i}}$, was calculated including the weightings above and $N_{i}$ is the number of sites surveyed in that year.

4. We fitted the following (generalised) linear model to assess the trend in the mean normalised peak count over time:

$$
\operatorname{glm}\left(K_{\text {norm }, i} \sim Y_{i}, \text { family }=\text { Gaussian, weights }=\left(1 / \alpha_{K_{\text {norm }, i}}\right)\right)
$$

where $Y_{i}$ is the year, we used a Gaussian error distribution since the model fitted the continuous mean normalised peak counts, and the weighting allowed the model more freedom where the uncertainty on the mean normalised peak count was large. A mean normalised peak count with uncertainty $2 \alpha$ therefore carried half as much weight in the model fit as a mean normalised peak count with uncertainty $\alpha$.

Having used the method above to determine comparable average population trends for both the small and large population sites, we also applied a more sensitive site-level analysis to the large population sites, since these did show well-sampled and roughly linear time series at site level. For these large population sites, we fitted a generalised linear model of the form: $\operatorname{glm}\left(k_{i, i} \sim Y_{i}+\right.$ factor $\left(\right.$ site $\left._{j}\right)$, family $=$ Poisson $\left(\right.$ link $={ }^{\prime} \log$ '), weights $\left.=w_{j, i}\right)$, where we used the peak counts from individual sites with a fixed site effect, Poisson errors (since we now use the un-normalised discrete counts), and weights set by $n_{\text {visits }}$ as described in step 2 above.

Where we identified declining population trends, we extrapolated the trend forward in time to estimate the number of years it would take for an average site to completely lose its adder population, assuming the populations continued to decline at their current rate, i.e. we calculated the year in which the mean normalised peak count was predicted to reach zero.

\subsubsection{Separation into Large and Small Population Sites}

We initially assumed $K_{\text {threshold }}=10$ and used the population trend results to test the suitability of using $K_{\text {threshold }}=10$ to separate the two groups. Calculating the average 
population trends for small and large population sites, as described in section 2.2.1, using $K_{\text {threshold }}=10$ revealed that the two groups show opposite average population trends. This suggests the optimum threshold at which to separate the two groups is that which produces the largest difference between their respective population trends. We assumed the large population sites would have above average mean peak counts. The average mean peak count across all sites included in the population trend analysis was 5.2. We therefore allowed the threshold to range in integer steps between $K_{\text {threshold }}$ $=5$ and $K_{\text {threshold }}=15$ (which leaves only six sites above the threshold) and recalculated the population trends for the small and large population sites using each threshold, to determine the most suitable value for $K_{\text {threshold }}$.

\subsubsection{Factors Affecting Sites}

We compared the average number of negative factors per site, average number of positive factors per site and average connectivity score per site for the small and large population sites. Due to non-normality of all six datasets, this was done by comparing medians using the MannWhitney U-Test for unmatched samples.

\subsubsection{Trends in Emergence Timing}

Surveyors reported the date of their site visits, allowing us to investigate variation between years in the date on which the peak count occurs. We defined the date on which the peak count occurs as the 'peak day' and we assumed variation in the peak day reflects variation in emergence timing for the majority of individuals.

In order to obtain peak day estimates that were as accurate as possible, we applied strict data selection criteria for this analysis; only peak day estimates that were obtained from five or more visits before day 153 (corresponding to 1st June in a leap year or 31st May in a non-leap year) were included. When the peak number of animals was recorded on more than one day, we used the earliest occurrence as the peak day.

We expect emergence timing to depend on spring temperatures and also site location. We therefore used the UK mean spring temperature time series produced by the Met Office (where spring is defined as the time period from March - May; downloaded from https://www.metoffice.gov.uk/climate/uk/summaries/ datasets\#yearOrdered; accessed 15 December 2017) and we regressed peak day against year, mean spring temperature $\left({ }^{\circ} \mathrm{C}\right)$, site latitude (described by each site's Ordnance Survey northing grid reference), site longitude (described by each site's Ordnance Survey easting grid reference) and the number of visits used to determine that peak day (to account for variation in surveyor effort) using a generalised linear model with a Poisson response and log link function, i.e. glm(PeakDay $\sim$ Year + SiteOSNorthing + SiteOSEasting + MeanSpringTemp + No.ofVisits, family $=$ Poisson $\left(\right.$ link $\left.\left.={ }^{\prime} \log ^{\prime}\right)\right)$. A Poisson response was used to account for the fact the peak day data are discrete, truncated at zero and left skewed.

We also calculated the mean peak day in each year across all the sites surveyed in that year and regressed this against the mean northing and mean easting of the sites surveyed in a given year, mean spring temperature and year, while controlling for the number of sites surveyed per year. This was done using a generalised linear model with a Gaussian response (since the response variable is now the yearly mean rather than discrete days) and log link, i.e. glm(MeanPeakDay $\sim$ Year + MeanOSNorthing + MeanOSEasting + MeanSpringTemp + No.ofSitesSurveyed, family = Gaussian $\left(\right.$ link $\left.\left.={ }^{\prime} \log ^{\prime}\right)\right)$. We then used step-wise reduction, eliminating the variable with the largest P-value in turn, to identify a model for mean peak day where all predictors were significant.

\section{Results}

From 2005 to 2016, 181 surveyors provided information on 260 sites. Figure 1 shows the geographical locations of these sites. More than $60 \%$ of the sites were described by their surveyors as being well connected to other adder populations (Fig. 2).

\subsection{Population Trends}

129 of the 260 sites had sufficient data (three or more years) to be included in the population trend analysis. Of these sites, 117 were classed as small population sites $(K \leq 10)$ and 12 were classed as large population sites $\left(K_{j}>10\right)$.

Table 1 contains descriptive statistics for the peak counts obtained from all sites qualifying for population trend analysis and for the small and large population sites separately. One site showed substantially higher peak counts $\left(K_{j}=94 \pm 5\right)$ than any of the other large population sites (next highest mean peak count among the large population sites was $K_{j}=32 \pm 1$ ), as can be seen by comparing the maximum peak counts in columns four and five of Table 1.

Figures $3 a$ and $b$ show the average populations trends for the small and large population sites, respectively, where the population trends were calculated as described by the steps in section 2.2.1.

The small population sites have shown a significant decline in their mean normalised peak count since the survey began (Fig. 3a). Fitting the generalised linear model described in step 4 of section 2.2.1, gave $K_{\text {norm }, i}=-(0.05 \pm 0.01) Y_{i}+(100 \pm 20)$, with $\mathrm{P}<0.01$ and $R^{2}=0.655$. 12 out of the 117 small population sites recorded no adders for the last two or more years those sites were surveyed, suggesting that just over $10 \%$ of these sites may potentially have lost their adder populations since the monitoring programme began. Extrapolating the trend in mean normalised peak count forward in time to estimate the number of years it would take for an average small population site to completely lose its adder population, assuming these small population sites continue to decline at their current rate, gave a lower limit estimate of just 16 years using the unrounded fit values. This implies the average small adder population will be extinct by 2032. We emphasise this is a lower limit estimate, as we are assuming that adders are absent when surveyors return zero counts and detectability is almost certainly not $100 \%$.

Figure $3 \mathrm{~b}$ shows the average population trend for 


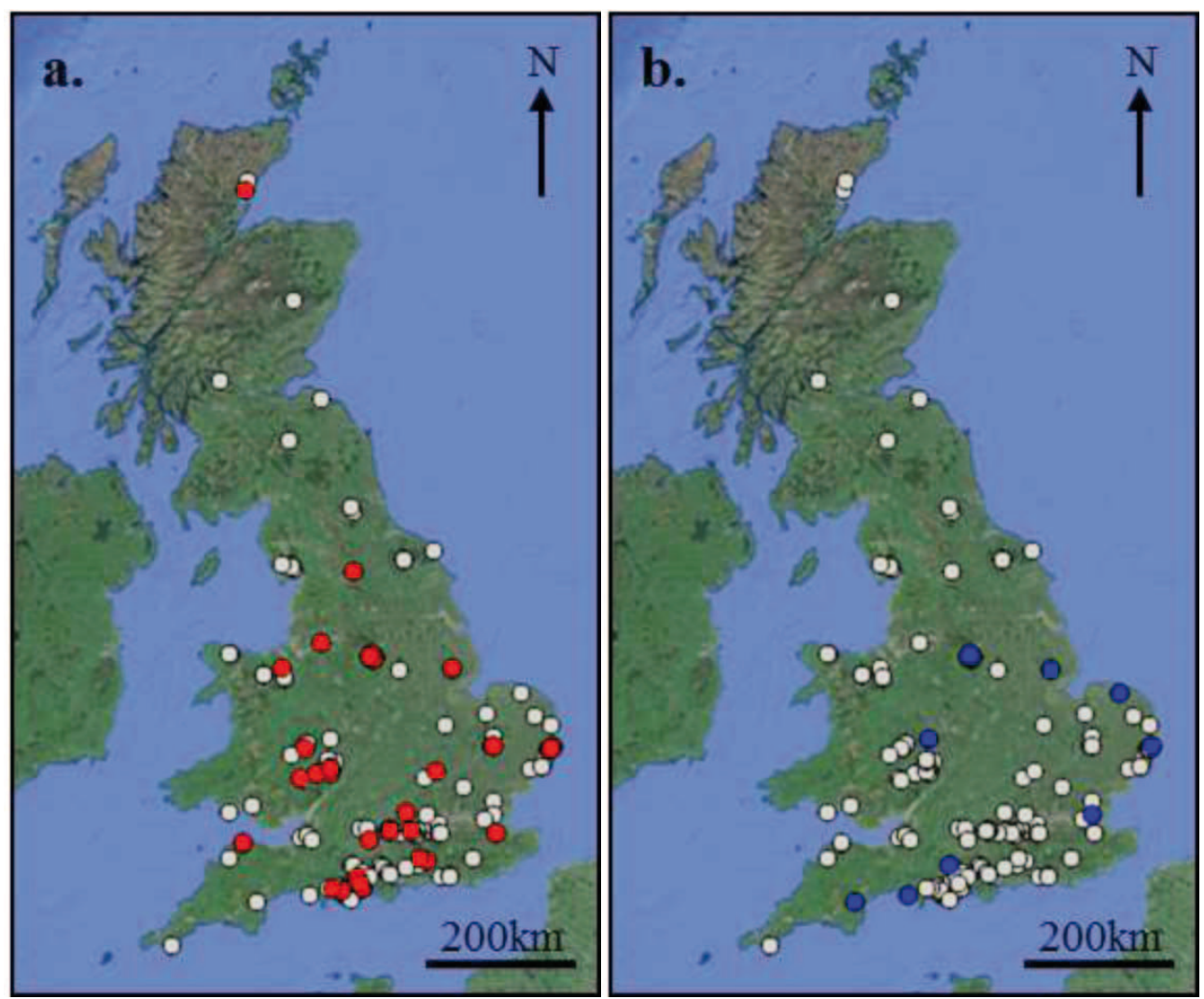

Figure 1. Geographical locations of survey sites. a) Red points show locations of small population sites included in the population trend analysis ( 117 sites with $K_{j} \leq 10$ and $3+$ years of data) and grey points show all other MTAC sites. b) Blue points show locations of large population sites included in the population trend analysis ( 12 sites with $K_{j}>10$ and $3+$ years of data) and grey points show all other MTAC sites. Note that some sites appear overlaid on the map due to their close proximity. Base maps from Google Satellite.

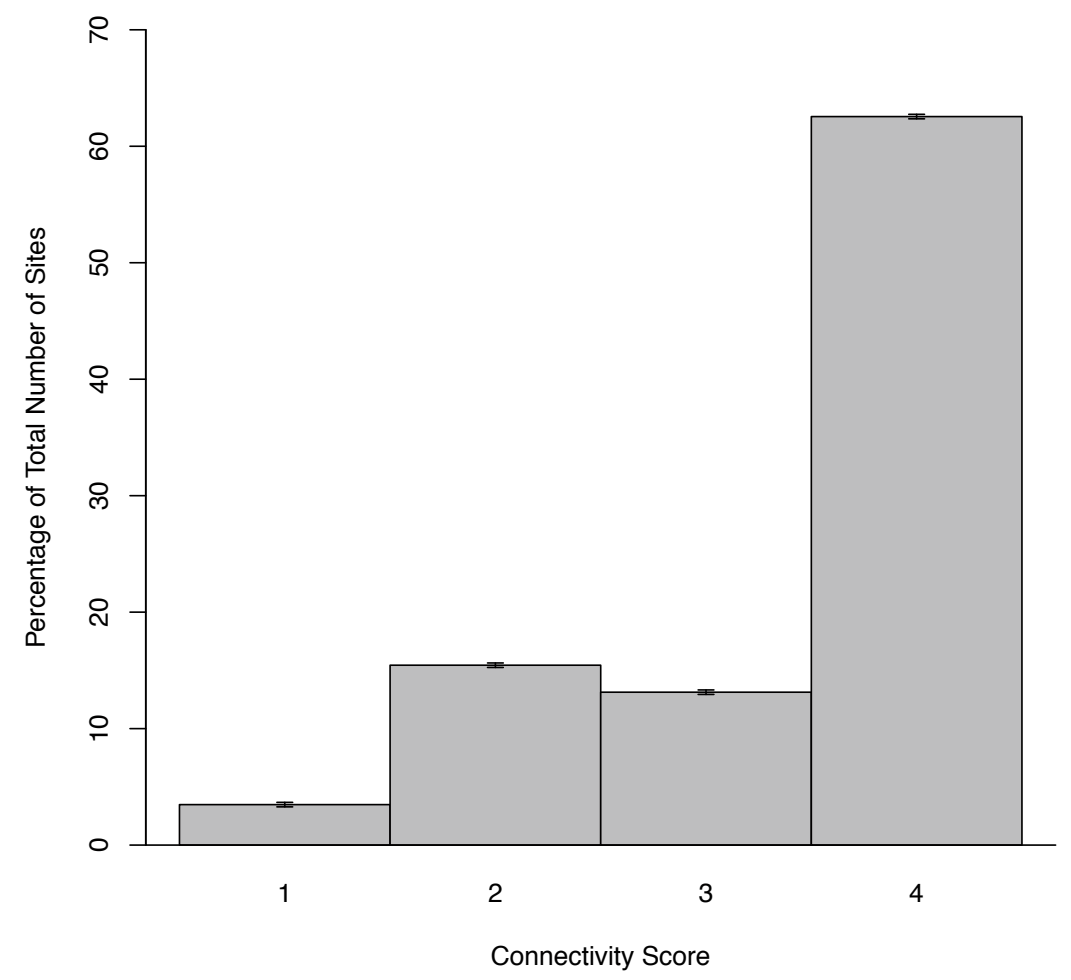

Figure 2. Connectivity scores reported for sites, where 1 = completely isolated by many kilometres, 2 = isolated from nearby sites by sub-optimal habitat, 3 = linked by corridors, 4 = part of a larger group of populations occupying more or less continuous habitat. Error bars show number of percentage points represented by a single survey site. 

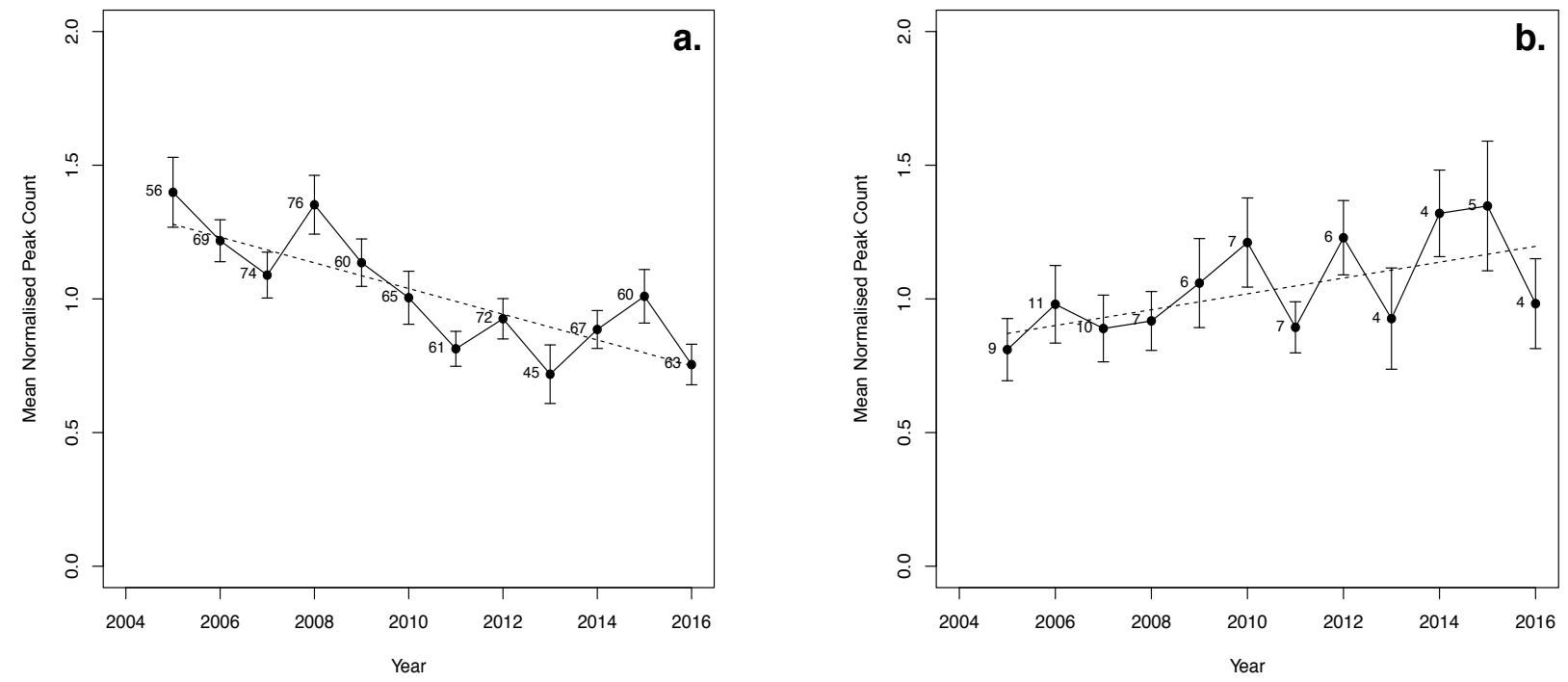

Figure 3. Average population trends for a) small population sites ( 117 sites with $K_{j} \leq 10$ and $3+$ years of data) and $\left.\mathbf{b}\right)$ large population sites (12 sites with $K_{r}>10$ and $3+$ years of data). Dashed lines show fits using the generalised linear model described in step 4 of section 2.2.1. Numbers adjacent to each data point show the number of normalised peak counts (i.e. the number of individual sites) contributing to the mean normalised peak count in that year. The number of sites contributing to each mean normalised peak count varies due to gaps in the time series from individual sites. Error bars show standard error on the mean, calculated as described in step 3 of section 2.2.1.

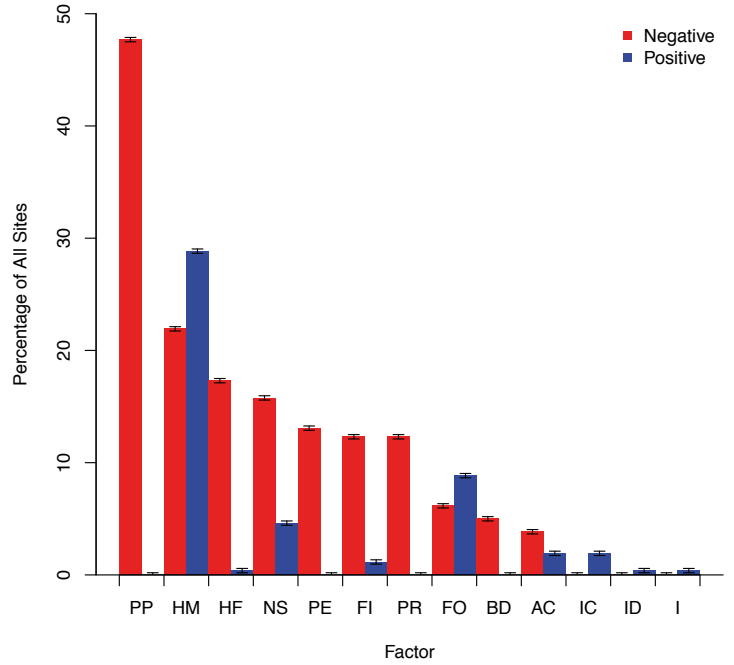

a.

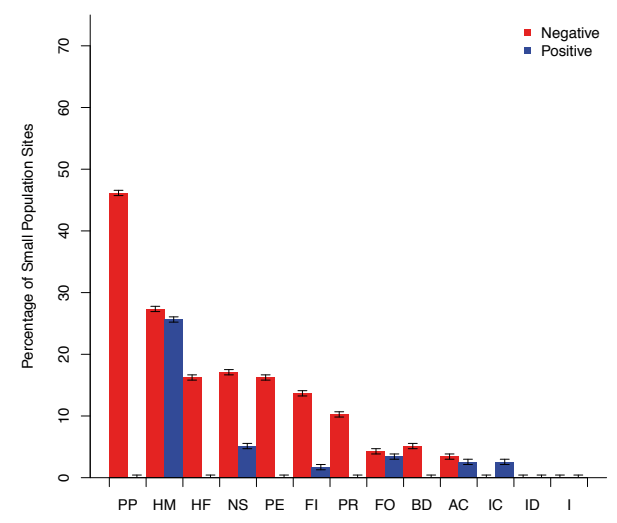

Factor
Figure 4. Percentage of all MTAC sites (260 sites) reported by surveyors to be positively and negatively affected by the following factors: PP = public pressure, $\mathrm{HM}=$ habitat management, $\mathrm{HF}=$ habitat fragmentation/isolation, $\mathrm{NS}=$ neglect/succession, $\mathrm{PE}=$ persecution, $\mathrm{FI}=$ fire, $P R=$ predation, $F O=$ forestry operations, $\mathrm{BD}=$ building development, $\mathrm{AC}=$ agricultural changes, IC = introduction (conservation), ID = introduction (development mitigation), I = introduction (unspecified). Error bars show number of percentage points represented by a single survey site.

b.

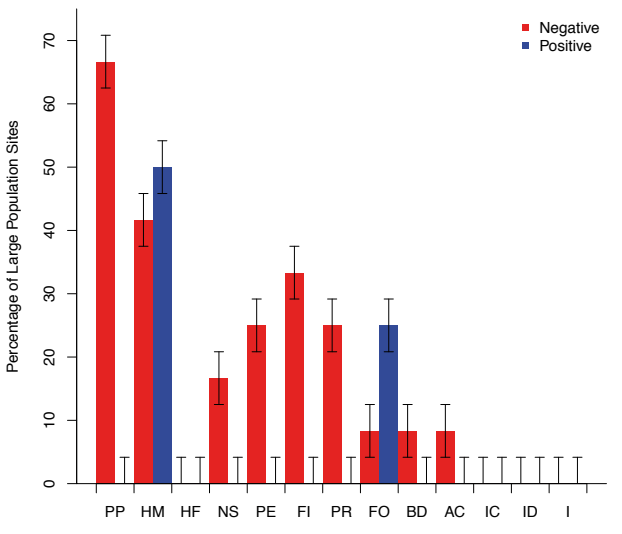

Factor

Figure 5. a) Factors affecting the small population sites included in the population trend analysis (117 sites with $K_{j} \leq 10$ and $3+$ years of data). b) Factors affecting the large population sites included in the population trend analysis (12 sites with $K_{i}>$ 10 and $3+$ years of data). Factor abbreviations: $\mathrm{PP}=$ public pressure, $\mathrm{HM}=$ habitat management, $\mathrm{HF}=$ habitat fragmentation/isolation, $\mathrm{NS}=$ neglect/succession, $\mathrm{PE}=$ persecution, $\mathrm{FI}=$ fire, $\mathrm{PR}=$ predation, $\mathrm{FO}=$ forestry operations, $\mathrm{BD}=$ building development, $\mathrm{AC}=$ agricultural changes, $\mathrm{IC}=$ introduction (conservation), ID = introduction (development mitigation), I = introduction (unspecified). Error bars show number of percentage points represented by a single survey site in each case. 
large population sites. In contrast, these have shown an increase in their mean normalised peak count. Fitting the generalised linear model described in step 4 of section 2.2.1, gave $K_{\text {norm }, i}=(0.03 \pm 0.01) Y_{i}-(60 \pm 30)$, with $\mathrm{P}<0.05$ and $\mathrm{R}^{2}=0.347$. For these large population sites, the trend was weaker and the $\mathrm{R}^{2}$ was lower. Since the large population sample was much smaller, we checked that the results were not unduly influenced by any one site (such as the outlying very large population site) by refitting the generalised linear model eliminating each site in turn from the dataset. We found the significance of the trend was not consistently robust to removal of individual sites from the model, although this was not due to any one site in particular, but rather due to a combination of the small sample size and the weakness of the trend. Fitting a more sensitive site-level model (gIm( $k_{j, i} \sim Y_{i}+$ factor(site $\left.)_{j}\right)$, family $=$ Poisson $\left(\right.$ link $={ }^{\prime} \log$ '), weights $=w_{j, i}$ ); see section 2.2.1) to these sites confirmed a significant positive trend $\left(k_{j, i} \propto(0.064 \pm 0.006) Y_{i}\right)$, with significance $\mathrm{P}<0.01$ which was consistently robust to removal of individual sites and showed a much improved $\mathrm{R}^{2}$ of 0.768 .

Finally, we tested the assumption that $K_{\text {threshold }}=10$ was a suitable threshold at which to separate the sites into large and small populations (see section 2.2.2) and found that the most negative trend in the small population group was obtained using $K_{\text {threshold }}=6$, while the most positive trend in the large population group was obtained using $K_{\text {threshold }}=12$. Given that the median of these two extremes is $K_{\text {threshold }}=9$, suggested that $K_{\text {threshold }}=10$ was a reasonable threshold to use for this dataset.

\subsection{Factors Affecting Sites}

In order to assess potential causes of population trends, surveyors were asked to report the positive and negative factors they considered were affecting their sites. Figure 4 shows the positive and negative factors reported across all MTAC sites. The most frequently reported factors positively affecting adder populations were habitat management ( $28 \%$ of sites), forestry operations (9\% of sites) and neglect/succession (5\% of sites). The most frequently reported negative factors were public pressure (48\% of sites), habitat management (22\% of sites) and habitat fragmentation/isolation (17\% of sites). Note that habitat management was reported as a negative factor almost as frequently as it was reported as a positive factor.

Figures $5 \mathrm{a}$ and $\mathrm{b}$ show separately the positive and negative factors reported for the small and large population sites included in the population trend analysis. Public pressure is still by far the greatest perceived threat in both cases, however there are notable differences in the reported impact of habitat management, forestry operations and habitat fragmentation. The small population sites reported habitat management and forestry operations as having both negative and positive effects, with slightly more sites reporting a negative effect. The large population sites similarly reported both negative and positive effects for these factors, but positive effects were reported more often than negative effects. None of the large population sites (which showed steady/increasing populations on average) reported habitat fragmentation as a factor, while $16 \%$ of the small population sites cited habitat fragmentation as a negative factor.

There was no significant difference between the median number of negative factors reported per site between the small and large population sites (MannWhitney U-test; $W=586, P=0.329$ ) nor in the median number of positive factors reported per site between the small and large population sites (Mann-Whitney U-test; $W=540, P=0.108)$. This suggested the declining small population sites were not simply facing more threats or experiencing fewer positive factors than the large population sites.

Table 2 compares the area classes, connectivity scores and conservation designations for the small and large population sites included in the population trend analysis. Caution should be used when comparing the percentages in Table 2, given the order of magnitude discrepancy in sample size between the small and large population sites. Nonetheless, there was no systematic difference between the area classes reported for the small and large population sites, with both showing roughly $15 \%$ of sites $\leq 5$ ha and $85 \%$ larger than 5 ha. There was also no significant difference between the median connectivity scores for the small and large population sites (MannWhitney U-test; $W=790, P=0.289$ ). However, the large population sites did include a higher proportion of SSSI and NNR designations and all of the large population sites had at least one conservation designation (SSSI, NNR, ONR, CWS or NP), whereas $15 \%$ of the small population sites had no conservation designation.

\subsection{Trends in Emergence Timing}

A total of 320 peak day estimates met the data selection criteria of five or more visits before day 153. Table 3 shows the results of regressing peak day against year, mean spring temperature, site latitude, site longitude and number of visits (as described in section 2.2.4). All four variables were found to be significant, with peak day generally becoming earlier over time and occurring earlier for higher mean spring temperatures and for more northerly and easterly sites. However, the $\mathrm{R}^{2}$ for this model was low (0.135) with much residual variance, likely due to site-specific factors unaccounted for in the modelling, such as site aspect, site shading and local micro-climatic weather variations.

Averaging over the individual sites to obtain the mean peak day per year should remove some of this additional variance. Regressing mean peak day per year against the mean northing of the sites surveyed in a given year, mean spring temperature and controlling for number of sites surveyed per year gave a much improved $R^{2}$ of 0.882 , with all three variables significant (Table 4). Neither year nor mean easting were found to be significant variables when fitting for mean peak day. The fitted coefficients implied a $\sim 11 \%$ decrease in peak day for a $100 \mathrm{~km}$ increase in northing and $\sim 5 \%$ decrease in peak day for a 1 degree increase in mean spring temperature. Using the mean peak day of 93 in 2005, these translate into real terms as $\sim 11$ day decrease in peak day for a $100 \mathrm{~km}$ increase in northing and $\sim 5$ day decrease in peak day for 
Table 1. Descriptive statistics for the peak counts obtained from all sites qualifying for population trend analysis (129 sites with $3+$ years of data), the subset of these which qualify as small population sites ( $K_{j} \leq 10 ; 117$ sites), the subset which qualify as large population sites $\left(K_{j}>10 ; 12\right.$ sites), and the subset of large population sites excluding the site with the highest mean peak count $\left(K_{j}=94 \pm 5\right)$ which is substantially higher than the mean peak counts of the other large population sites.

\begin{tabular}{|c|c|c|c|c|}
\hline Peak Count Statistic & $\begin{array}{l}\text { All sites qualifying for } \\
\text { population trend analysis }\end{array}$ & $\begin{array}{c}\text { Small } \\
\text { population sites }\end{array}$ & $\begin{array}{c}\text { Large } \\
\text { population sites }\end{array}$ & $\begin{array}{l}\text { Large population sites } \\
\text { excluding outlier }\end{array}$ \\
\hline Minimum & 0 & 0 & 1 & 1 \\
\hline 1st Quartile & 1 & 1 & 10 & 10 \\
\hline Median & 3 & 3 & 14.5 & 14 \\
\hline Mean & $5.66 \pm 0.01$ & $3.626 \pm 0.004$ & $25.2 \pm 0.4$ & $15.3 \pm 0.1$ \\
\hline 3rd Quartile & 6 & 5 & 23 & 18.75 \\
\hline Maximum & 167 & 19 & 167 & 38 \\
\hline Total No. of Peak Counts & 848 & 768 & 80 & 70 \\
\hline
\end{tabular}

Table 2. Comparison of site characteristics for small population sites included in the population trend analysis (117 sites with $K_{j} \leq 10$ and $3+$ years of data) with site characteristics for large population sites included in the population trend analysis (12 sites with $K_{j}>10$ and $3+$ years of data). Connectivity scores: $1=$ completely isolated by many kilometres, $2=$ isolated from nearby sites by sub-optimal habitat, 3 = linked by corridors, 4 = part of a larger group of populations occupying more or less continuous habitat. Conservation designations: SSSI = site of special scientific interest, NNR = national nature reserve, ONR = other nature reserve, CWS = community wildlife site, NP = national park, NCD = no conservation designation. Sites may have more than one conservation designation.

\begin{tabular}{lccc}
\hline & & $\begin{array}{c}\text { Percentage (\%) of small population } \\
\text { sites }\end{array}$ & $\begin{array}{c}\text { Percentage (\%) of large population } \\
\text { sites }\end{array}$ \\
\hline \multirow{3}{*}{ Area Class } & 5 ha & 13 & 17 \\
& 5 ha & 84 & 83 \\
Connectivity Score & NA & 3 & 0 \\
& 1 & 3 & 0 \\
& 2 & 13 & 17 \\
Conservation Designation & 3 & 9 & 25 \\
& 4 & 74 & 58 \\
& NA & 2 & 0 \\
& SSSI & 62 & 83 \\
NNR & 4 & 17 \\
ONR & 16 & 8 \\
& CWS & 15 & 33 \\
& NP & 40 & 0 \\
\hline
\end{tabular}

Table 3. Results from fitting the generalised linear model PeakDay Year + SiteOSNorthing + SiteOSEasting + MeanSpringTemp + No.ofVisits (family $=$ Poisson, link $=\log$ ) using site level data. Model $R^{2}=0.135$. Asterisks indicate statistically significant correlations, where $*=\mathrm{P}<0.05, * *=\mathrm{P}<0.01$ and $* * *=\mathrm{P}<0.001$.

\begin{tabular}{|c|c|c|c|}
\hline Variable & Estimate & Std. Error & Significance \\
\hline Intercept & 29 & 4 & $* * *$ \\
\hline Year & $-1.2 \times 10^{-2}$ & $0.2 \times 10^{-2}$ & $* * *$ \\
\hline Site OS Northing & $-1.7 \times 10^{-7}$ & $0.5 \times 10^{-7}$ & $* * *$ \\
\hline Site OS Easting & $-1.3 \times 10^{-7}$ & $0.6 \times 10^{-7}$ & $*$ \\
\hline Mean Spring Temp. & $-7.7 \times 10^{-2}$ & $1.0 \times 10^{-2}$ & $* * *$ \\
\hline No. of Visits & $7 \times 10^{-3}$ & $2 \times 10^{-3}$ & $* * *$ \\
\hline
\end{tabular}


a 1 degree increase in mean spring temperature.

Surveyors were asked, where possible, to record male and female adders separately and this allowed us to investigate differences between the peak day for males and the peak day for females. We applied the same strict selection criteria and only included peak day estimates obtained from five or more visits recording male and female adders separately before day 153 . Not all surveys returned results for males and females separately, either because surveyors were not confident separating them or because the conditions on a given survey were not conducive to reliably distinguishing the sexes by sight and (often) at a distance. Consequently, the number of male peak day and female peak day estimates (238 and 131 estimates, respectively) were lower than the number of peak day estimates using the total number of animals. Figure 5 shows the resulting frequency distributions for male peak day estimates (blue) and female peak day estimates (red), where the estimates are binned on fortnightly intervals. Figure 5 shows that the male peak day distribution peaks a fortnight earlier than the female peak day distribution.

\section{Discussion}

The population trend analysis shows that, on average, the small population sites have shown a significant decline in their peak counts over time $\left(K_{n o r m, i} \propto-(0.05 \pm 0.01)\right.$ $\left.Y_{i}\right)$, while the large population sites have shown a significant, but slightly weaker, increase over time $\left(K_{n o r m, i} \propto(0.03 \pm 0.01) Y_{i}\right)$. This confirms earlier informed opinion that small populations are more prone to decline than large populations (Baker et al., 2004). More than 90 $\%$ of the sites included in the population trend analysis fell into the small (declining on average) population category, with only $12 / 129$ sites being classed as large (increasing on average) population sites $\left(K_{j}>10\right)$. If these proportions and trends are representative of UK adder sites in general, then the results from MTAC suggest that adders may become increasingly restricted to a few large population sites, making the species increasingly vulnerable to extinction in the UK. The rate at which these small population sites are declining implies the average small adder population could be extinct by 2032 .

The declines we have measured in UK adder populations are consistent with the wider picture of snake declines shown by Reading et al. (2010), who showed that 11 out of 17 snake populations spread across Europe, Nigeria and Australia have declined over a 20 year period. Saha et al. (2018) subsequently

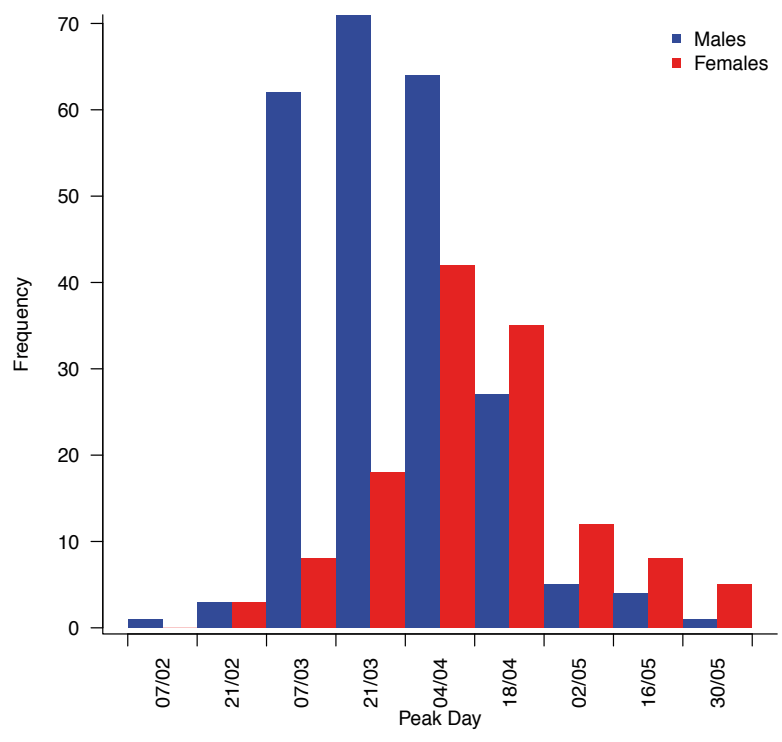

Figure 6. Frequency distributions for male peak day estimates (blue) and female peak day estimates (red), where the estimates are binned in fortnightly intervals. For each bin, the date (day/month) given is the midpoint of that bin.

analysed data from the Living Planet database to show that such global declines are not confined to snakes, but shown by the entire reptile taxon. Both studies stressed the importance, not only of monitoring reptile declines, but also of collecting auxiliary data to identify potential causes, as we have attempted to do via MTAC.

We found no systematic difference between the area classes reported for the declining small population sites and the increasing large population sites. This suggests that the small population sites have sufficient area to support larger populations but habitat quality or some other factor is depressing the population, or that the wider site around them potentially contains other hibernacula which may or may not be included in MTAC. There was also no significant difference between the median connectivity scores for the small and large population sites. However, despite this, none of the large population sites reported habitat fragmentation as a negative factor, while $16 \%$ of the small population sites did. The declines seen in the small population sites are consistent with inbreeding depression, as seen in an isolated Swedish adder population, where reduced genetic diversity, smaller brood sizes and reduced offspring viability (Madsen \& Shine, 1996) have been linked to a population decline (Madsen et al., 1999). This suggests small population sizes and increased habitat

Table 4. Results from fitting the generalised linear model MeanPeakDay $\sim$ MeanOSNorthing + MeanSpringTemp + No.ofSitesSurveyed (family = Gaussian, link $=\log$ ) using data averaged over all sites surveyed in a given year. Model $R^{2}=0.882$. Asterisks indicate statistically significant correlations, where $*=\mathrm{P}<0.05, * *=\mathrm{P}<0.01$ and $* * *=\mathrm{P}<0.001$.

\begin{tabular}{lccl}
\hline Variable & Estimate & Std. Error & Significance \\
\hline Intercept & 5.2 & 0.2 & $* * *$ \\
Mean OS Northing & $-1.3 \times 10^{-6}$ & $0.3 \times 10^{-6}$ & $* *$ \\
Mean Spring Temp. & $-6 \times 10^{-2}$ & $2 \times 10^{-2}$ & $* *$ \\
No. of Sites Surveyed & $2.4 \times 10^{-3}$ & $0.9 \times 10^{-3}$ & $*$ \\
\hline
\end{tabular}


fragmentation may be combining to promote inbreeding and further declines at some of these sites. Work is currently ongoing to quantify genetic diversity across a sample of UK sites and its role in relation to other factors (S. Ball, T. Garner \& N. Hand, personal comm.).

There was no significant difference between the large and small population sites in the median number of negative factors reported per site, nor in the median number of positive factors reported per site. However, there were more positive than negative reports for habitat management and forestry operations for the large population sites, while for the small population sites, negative reports outweighed positive reports for both factors. The large population sites included a higher proportion of SSSI and NNR designations and all of the large population sites had at least one conservation designation (SSSI, NNR, ONR, CWS or NP), whereas 15 $\%$ of the small population sites had no conservation designation. This may have contributed towards more positive habitat management and forestry operation outcomes in the large population sites and the fact that neglect/succession was the third biggest negative factor reported for the small population sites. However, simply having a conservation designation clearly does not guarantee positive management impacts for adders. This conclusion is not unique to the UK; Reading et al. (2010) likewise found that eight snake populations had declined out of the 14 within their sample that were located within protected areas.

Across all the MTAC sites, by far the most frequently reported negative factor was public pressure $(48 \%$ of sites), followed by habitat management (22\% of sites) and habitat fragmentation/isolation (17\% of sites). Comparing these top three negative factors with those reported in previous studies (Cooke \& Scorgie, 1983; Hilton-Brown \& Oldham, 1991; Baker et al., 2004) indicates some notable changes in the perceived importance of factors over time. There has been a reduction in the perceived importance of agricultural changes (no longer in the top three in Baker et al., 2004 or MTAC), with high citation of agricultural changes in the earlier studies likely reflecting the intensification of agriculture during the 1970 s-80s. Persecution also entered the top three in two out of the three previous studies, but is ranked fifth in MTAC. Habitat fragmentation is the third most cited negative factor in MTAC but was not even included as a category in the earlier studies and this probably reflects both an increase in the importance of this factor as well as increasing awareness of its significance. Negative reporting of public pressure and habitat management (the top two negative factors in MTAC) has increased dramatically across the four studies. Where public pressure was reported to MTAC, surveyors variously cited dog walking, mountain biking, photographers, vehicles, trampling of vegetation and collection of adders as causing problems at sites. There is very little quantitative data relating to the effects of disturbance on reptiles and the dramatic increase in the reporting of public pressure, such that nearly half of sites are now negatively affected by public pressure, suggests this factor needs urgent investigation, both to assess its impacts on adder populations and to identify potential measures to reduce these impacts.

The most frequently reported factors positively affecting adder populations were habitat management (28\% of sites), forestry operations (9\% of sites) and neglect/succession (5\% of sites). Where neglect/ succession was cited as a positive factor, this was often for sites recovering after earlier aggressive habitat management. Adders have complex habitat requirements, using both open areas for basking and cover for hunting and travelling. Jofré et al. (2016) showed reptiles in conifer plantations prefer stands between $3-12$ years old, suggesting that managing woodlands to produce a mosaic of small stands of different ages could increase the proportion of sites benefitting from forestry operations. While habitat management was the largest positive factor reported, the fraction of sites experiencing positive effects from habitat management ( $28 \%$ ) only just outweighed the proportion experiencing negative effects ( $22 \%$ ), i.e. where habitat management occurred, only in $\leq 56 \%$ of cases was it considered to have a positive effect on adders. Examples of negative effects of habitat management given by the surveyors included overgrazing, heavy machinery/mechanical cutting, bracken clearance and managing specifically for another species with different habitat requirements (also noted by Sheldon, 2011). This suggests greater awareness is needed among land managers of adders' habitat requirements and of the threats posed by heavy machinery, particularly during hibernation and the spring emergence period. Advice on habitat management is available (Edgar et al., 2010) but our findings suggest that it requires better communication and/or implementation.

The date that adder counts peaked at the sites varied significantly over time and with spring temperature, site northing and site easting grid references. Peak counts were earlier under higher mean spring temperatures $(\sim 5$ day decrease in peak day for a 1 degree increase in mean spring temperature) and occurred approximately 10 days earlier by the end of the eleven-year data collection period. Peak counts also occurred earlier at more easterly and northerly sites ( $\sim 11$ day decrease in peak day for a $100 \mathrm{~km}$ increase in northing). Earlier emergence for warmer temperatures is expected for ectotherms such as adders and has also been noted by Viitanen (1967) and Macartney et al. (1989). The advancement in peak day over time found in the site-level model fit may be a response to long-term climate warming, similar to the advancement in amphibian breeding behaviour in Britain (e.g. Beebee, 1995; Scott et al., 2008; Carroll et al., 2009), although we detected no significant warming trend in spring or winter temperatures during our (shorter) monitoring period. Alternatively, given the geographical trends in emergence timing, it may simply be an artefact of spatial variation in temporal coverage. It is unclear why adders should emerge earlier at more northerly sites; however this has been noted anecdotally by some surveyors. One possibility may be that lower temperatures and fewer total sunshine hours at northern sites may mean adders need to bask over a longer period to gain the same total amount of thermal energy. 
Alternatively, adders at northern sites may simply be better adapted to lower temperatures so allowing them to emerge earlier. At least one invertebrate (glow worm, Lampyris noctiluca) shows a similar trend of earlier adult emergence at more northerly sites in the UK (Atkins et al., 2016; J.Tyler personal comm.). Although all four variables (year, mean spring temperature, northing and easting) were significant in the site-level model, there was still large residual variation in emergence timing across the sites and this likely reflects a number of sitespecific factors unaccounted for in the modelling, such as site aspect, site shading and regional weather variations. Hibernacula with southerly aspects should warm faster, producing earlier emergence, while high site shading by trees and other vegetation may delay warming and emergence. Blouin-Demers et al. (2000) similarly observed large variation in emergence timing between different black rat snake hibernacula and found larger snakes emerge earlier, possibly due to lower predation risk. This suggests variations in predation risk and the size distributions of adders between hibernacula may also be a contributing factor. Surveyor activity (i.e. exact timing of visits) is another large and unavoidable source of variation, though we attempted to minimise this as much as possible by requiring five or more visits before the start of June and controlling for number of visits in the model.

The trends in emergence timing have important implications for habitat management. If hibernacula can be located, they can be avoided when carrying out habitat management using heavy machinery and operations can be timed to avoid the emergence period. The majority of peak counts were recorded from $\sim 1$ st March onwards, however adders begin emerging several weeks before the peak count is reached, so a general cut-off of 1st February for management operations may be appropriate. However, managers should be aware that adders may emerge $\sim 2-3$ weeks earlier in a warm spring versus a cold spring (based on a 3 degree difference between the maximum and minimum mean spring temperatures during this study of $6^{\circ} \mathrm{C}$ and $9^{\circ} \mathrm{C}$ ) and management operations at more northerly/easterly sites may need to be completed earlier.

We found that the peak counts of male adders occurred $\sim 2$ weeks earlier than the peak counts for female adders. This is consistent with the earlier emergence of males noted in other studies (Prestt, 1971; Phelps, 2008), with this early basking behaviour corresponding to a period of active spermiogenesis (Viitanen, 1967; Prestt, 1971; Herczeg et al., 2007). The fact our study included nearly twice as many peak day estimates for males as for females is a measure of the bias in the survey methodology towards detection of these early basking males in preference to the later emerging females. Sheldon (2011) found that non-breeding females not only emerge later than males but also disperse more quickly from hibernacula and that females in the Wyre Forest bred on average every three years, such that the number of females observed in spring counts may only represent the fraction of females that are breeding in any given year.
MTAC has demonstrated the potential of spring counts made by volunteer surveyors to collect quantitative data on adder populations, which can be used to derive population trends. To date, the scheme has relied on experienced surveyors. However, the skill level required is comparable to that required for other skilled citizen science surveys operating in the UK, such as the UK Breeding Bird Survey (organised by the British Trust for Ornithology; Baillie et al., 2006) and the National Bat Monitoring Programme (organised by the Bat Conservation Trust; Barlow et al., 2015), which both provide regional training to new volunteers to help them attain the required experience. If such training schemes can be developed for MTAC, this would greatly increase the volunteer pool available and the number of sites that can be monitored.

\subsection{Caveats and Assumptions}

We have assumed that changes in the number of adders recorded at a given site reflect genuine changes in population size and are not influenced by systematic changes in their detectability over time. Our analysis accounts for variation in survey effort, which strongly influences snake detectability (Ward et al., 2017), but other factors may also influence detectability. Detectability may increase over time if surveyors become more familiar with a site. However, since MTAC specifically targeted experienced surveyors with good existing knowledge of adder sites, we assume this effect is negligible. Habitat changes may systematically alter detectability at a site, e.g. if increasing vegetation reduces adder visibility over time. The fraction of sites negatively affected by neglect/succession (i.e. where increasing vegetation might potentially reduce adder detectability) was similar for both the large and small population sites, so changes in detectability are unlikely to explain the differing trends for large and small population sites.

We have assumed that detectability is comparable between sites, such that we can equate sites with large mean peak counts to large population sites and sites with small mean peak counts to small population sites. The fact that we derived a declining population trend for the subset of small population sites and an increasing population trend for the subset of large population sites suggests that this separation is plausible. It is possible that some of the small population sites were misclassified large population sites with low detectability. We currently have no information on the habitat types and vegetation densities at each site. If this data can be collected, then an improved population trend model incorporating this information to account for detectability differences between sites (and potentially over time) would improve population trend estimates and increase confidence in the division of sites into small and large populations.

We have assumed the measured declines at small population sites represent a genuine decrease in the number of adders, not that the adders have simply moved elsewhere, and that sites are independent. Adders are highly site faithful with relatively small home ranges ( $<5$ ha; Langton \& Beckett, 1995), but nonetheless, this independence assumption may not 
be true for sites that are very close together. Just over $80 \%$ of the small population sites and $50 \%$ of the large population sites are within $1 \mathrm{~km}$ of another MTAC site, with a mean minimum distance of $2.4 \mathrm{~km}$ to the next nearest MTAC site for small population sites versus 13.6 $\mathrm{km}$ for the large population sites. We stress that these minimum distances are approximate, given that $58 \%$ of sites supplied grid references at $100 \mathrm{~m}$ (three figure) resolution or less. These minimum distances are in good agreement with surveyors reporting the majority of sites to be well connected to other adder populations and suggest it is feasible that adders could move from one site to another, with this potentially more likely among the small population sites. However, since we derive average population trends, if adders move from one site to another, the decrease at one site should be offset by an increase at the other, assuming surveyors are aware of and surveying all hibernacula in the vicinity. The scheme's targeting of experienced surveyors with good prior knowledge of their sites increased the chances of this being the case and there were a few instances where surveyors stopped surveying a site that had gone to zero counts and started surveying another that was very nearby, suggesting this may occasionally happen.

Figure 1 shows that the geographical coverage of MTAC is currently strongly biased towards southern sites and this bias is further exacerbated by the data quantity restrictions required for inclusion in the population trend analysis. Although there are far fewer large population sites, figure 1 shows that these are reasonably well distributed across the geographical range of the small population sites. Work began in 2018 to recruit more surveyors and encourage resurveying of existing sites, in order to reduce this geographical bias and increase the confidence with which MTAC data can be used to represent UK adder population trends in general.

We have identified factors potentially affecting adder populations, however, as in previous studies (Cooke \& Scorgie, 1983; Hilton-Brown \& Oldham, 1991; Baker et al., 2004), these are the factors perceived by surveyors to be important. As such, there may be a bias towards those factors (e.g. public pressure) that leave clear signs visible to surveyors, while factors which are less visible (e.g. prey limitation, predation) may be underreported. Fluctuations in prey numbers have been shown to limit population size in an isolated adder population (Forsman \& Lindell, 1997), while predation by pheasants may severely reduce reptile populations, particularly where pheasant densities are high (Rice, 2016), although much of the evidence on the impacts of pheasant predation remains anecdotal due to a lack of quantitative studies of sufficient size (Dimond et al., 2014). Changes in prey abundance are unlikely to be apparent to MTAC surveyors and the number of dead or injured adders encountered by surveyors may not accurately reflect predation pressure. We stress that detailed field studies are needed to truly assess the relative importance of such factors in relation to those highlighted in this study.

\section{Conclusions}

Make the Adder Count (MTAC) began in 2005 as a citizen science scheme designed specifically to gather the quantitative data needed to monitor adder population trends in the UK, to assess current threats to adder populations and to inform conservation actions. Surveyors were asked to make three or more counts each spring of adult adders lying out after emerging from hibernation, and to report the positive and negative factors they considered were affecting their adder sites. Between 2005 and 2016, 181 surveyors provided information on 260 sites. 129 of these sites contributed three or more years' worth of data and these were used to derive average population trends over time.

The data indicate a significant decline, on average, across sites with small adder populations $\left(K_{j} \leq 10\right.$ individuals), while the relatively few sites with large adder populations ( $<10 \%$ of sites) showed a significant increase over time. If these trends are representative of the UK as a whole, this suggests adders may become increasingly restricted to a few large population sites. The rate at which the small population sites are declining implies an average small adder population could be extinct by 2032, putting a timescale on this process of just $10-15$ years. This restriction of adders to a few large population sites will greatly increase the extinction risk for this priority species in the UK.

Analysis of the factors reported to be affecting sites highlights three key factors which must be addressed, if we are to halt the observed decline in adder numbers in the UK:

1. Public pressure through disturbance (most frequently reported threat; $48 \%$ of sites). The fact that public pressure was reported to negatively affect almost half of the 260 sites suggests this is an issue which needs urgent attention, to quantify its effects on adders and, if necessary, to identify effective means of reducing this pressure.

2. Habitat management (most frequently reported positive factor $-28 \%$ of sites - and second most frequently reported negative factor $-22 \%$ of sites). Greater effort must be made to raise awareness among land managers of the habitat requirements and activity patterns of adders. Currently around half of management operations negatively impact adders; if these negative impacts can be replaced by positive impacts, e.g. by protecting hibernacula and avoiding use of heavy machinery during active periods, this could contribute significantly towards halting population declines.

3. Habitat fragmentation (third most frequently reported negative factor; $17 \%$ of sites). Just over $80 \%$ of the small population sites were $<1$ $\mathrm{km}$ from another site and $16 \%$ of them reported habitat fragmentation as a negative factor, while none of the large population sites were reportedly affected by habitat fragmentation. This suggests maintaining connectivity, especially between small populations, should be a priority.

MTAC has shown that spring counts by volunteer surveyors are a viable method for collecting quantitative data on adder populations, and not only can these data quantify population trends, but they can also provide 
insights into adder behaviour and phenology. The current dataset demonstrates the earlier emergence of males and reveals that adder emergence occurs earlier, not only for warmer springs, but also for more northerly sites. The MTAC database provides an invaluable record of hibernacula locations across the UK and current effort now focuses on improving the geographical coverage of the survey, both by encouraging resurveying of existing sites and recruiting new volunteers. We hope that the declines shown by MTAC may motivate landowners and organisations to act to conserve adders and their habitat, that conservation interventions can be developed which address the three key threats identified in the survey, and that MTAC itself may grow and continue to provide the means to monitor the effectiveness of such interventions.

\section{ACKNOWLEDGEMENTS}

MTAC has had several financial supporters over the years including Amphibian and Reptile Conservation, Derbyshire ARG, Natural England, Habitat Aid and Herpetologic Ltd. The current analysis has been coordinated by ARG UK with financial support from the Clark Bradbury Charitable Trust and the Patsy Wood Trust. We thank R.Griffiths, R.Ward and W.Wüster for helpful conversations during manuscript preparation and two reviewers for detailed and constructive comments. We thank the 181 volunteer surveyors who contributed data and without whom this work would not have been possible.

\section{REFERENCES}

Arnold, H.R. (1995). Atlas of Amphibians and Reptiles in Britain. ITE Research Publication No. 10, HMSO.

Atkins, V., Bell, D., Bowker, A., Charig, M., Crew, J., Dale, M., Hickmott, B., Payne, B., Pendleton, D., Pendleton, T., Robinson, M., Wollen, K., Woodell B. \& Tyler, J. (2016). The status of the glow-worm Lampyris noctiluca L. (Coleoptera: Lampyridae) in England. Lampyrid 4, 20-35.

Baillie, S.R., Marchant, J.H., Crick, H.Q.P., Noble, D.G., Balmer, D.E., Coombes, R.H., Downie, I.S., Freeman, S.N., Joys, A.C., Leech, D.I., Raven, M.J., Robinson, R.A. \& Thewlis, R.M. (2006). Breeding Birds in the Wider Countryside: Their Conservation Status 2005. BTO, Thetford.

Baker, J., Suckling, J. \& Carey, R. (2004). Status of the adder Vipera berus and slow-worm Anguis fragilis in England. English Nature Research Reports Number 546. Peterborough: English Nature, 44 .

Barker, R.J., Schofield, M.R., Link, W.A. and Sauer, J.R. (2018). On the reliability of $\mathrm{N}$-mixture models for count data. Biometrics 74(1), 369-377.

Barlow, K.E., Briggs, P.A., Haysom, K.A., Hutson, A.M., Lechiara, N.L., Racey, P.A., Walsh, A.L. \& Langton, S.D. (2015). Citizen science reveals trends in bat populations: the National Bat Monitoring Programme in Great Britain. Biological Conservation 182, 14-26.

Beebee, T.J.C. (1995). Amphibian breeding and climate. Nature 374, 219-220.

Beebee, T.J.C. \& Griffiths, R. A. (2000). Amphibians and Reptiles. A Natural History of the British Herpetofauna. London: Harper Collins, 270.
Blouin-Demers, G., Prior, K.A. \& Weatherhead, P.J. (2000). Patterns of variation in spring emergence by black rat snakes (Elaphe obsoleta obsoleta). Herpetologica, 175-188.

Carroll E.A., Sparks, T.H., Collinson, N. \& Beebee, T.J.C. (2009). Influence of temperature on the spatial distribution of first spawning dates of the common frog (Rana temporaria) in the UK. Global Change Biology 15, 467-473

Cooke, A.S. \& Arnold, H.R. (1982). National changes in the status of the commoner British amphibians and reptiles before 1974. British Journal of Herpetology 6, 206-207.

Cooke, A.S. \& Scorgie, H.R.A. (1983). The status of the commoner amphibians and reptiles in Britain. Focus on Nature Conservation, No. 3. Peterborough: Nature Conservancy Council.

Dimond, R., Wheeler, M., Hand, N. \& Westbury, D. (2014). An investigation into the relationship between pheasants (Phasianus colchicus) and reptiles as prey. Conference Report 2013. Herpetological Journal 24, 3-6.

Edgar, P., Foster, J. \& Baker, J. (2010). Reptile Habitat Management Handbook. Bournemouth: Amphibian and Reptile Conservation.

Forsman, A. \& Lindell, L.E. (1997). Responses of a predator to variation in prey abundance: survival and emigration of adders in relation to vole density. Canadian Journal of Zoology 75(7), 1099-1108.

Gleed-Owen, C. \& Langham, S. (2012). The Adder Status Project - a conservation condition assessment of the adder (Vipera berus) in England, with recommendations for future monitoring and conservation policy. Unpublished report. CGO Ecology Ltd, Bournemouth.

Herczeg, G., Saarikivi, J., Gonda, A., Peraelae, J., Tuomola, A. \& Merilae, J. (2007). Suboptimal thermoregulation in male adders (Vipera berus) after hibernation imposed by spermiogenesis. Biological Journal of the Linnean Society 92(1), 19-27.

Hilton-Brown, D. \& Oldham, R.S. (1991). The status of the widespread amphibians and reptiles in Britain, 1990, and changes during the 1980s. Focus on Nature Conservation, No. 131. Peterborough: Nature Conservancy Council.

Jofré, G.M., Warn, M.R. \& Reading, C.J. (2016). The role of managed coniferous forest in the conservation of reptiles. Forest Ecology and Management 362, 69-78.

Langton, T.E.S. \& Beckett, C.L. (1995). Home range size of Scottish amphibians and reptiles. Scottish Natural Heritage Review No. 53.

Macartney, J.M., Larsen, K.W. \& Gregory, P.T. (1989). Body temperatures and movements of hibernating snakes (Crotalus and Thamnophis) and thermal gradients of natural hibernacula. Canadian Journal of Zoology 67(1), 108-114.

Madsen, T. \& Shine, R. (1992). Sexual competition among brothers may influence offspring sex ratio in snakes. Evolution 46(5), 1549-1552.

Madsen, T., Stille, B. \& Shine, R. (1996). Inbreeding depression in an isolated population of adders Vipera berus. Biological Conservation 75, 113-118.

Madsen, T., Shine, R., Olsson, M. \& Wittzell, H. (1999). Restoration of an inbred adder population. Nature 402, 34-35.

Phelps, T. (2004). Population dynamics and spatial distribution of the adder Vipera berus in southern Dorset, England. Mertensiella 15, 241-258. 
Phelps, T. (2008). Changes in the phenology of the adder, Vipera berus, over four decades: a comparison with Prestt (1971). The Herpetological Bulletin 103, 32-36.

Prestt, I. (1971). An ecological study of the viper Vipera berus in southern Britain. Journal of Zoology 164(3), 373-418.

QGIS Development Team (2015). QGIS Geographic Information System. Open Source Geospatial Foundation Project. https://qgis.org

R Core Team (2017). R: A language and environment for statistical computing. R Foundation for Statistical Computing, Vienna, Austria. URL https://www.R-project.org/.

Reading, C.J., Buckland, S.T., McGowan, G.M., Jayasinghe, G., Gorzula, S. \& Balharry, D. (1996). The distribution and status of the adder (Vipera berus L.) in Scotland determined from questionnaire surveys. Journal of Biogeography 23(5), 657-667.

Reading, C.J., Luiselli, L.M., Akani, G.C., Bonnet, X., Amori, G., Ballouard, J.M., Filippi, E., Naulleau, G., Pearson, D. \& Rugiero, L. (2010). Are snake populations in widespread decline? Biology Letters 6(6), 777-780.

Rice, C.N. (2016). Abundance, impacts and resident perceptions of non-native common pheasants (Phasianus colchicus) in Jersey, UK Channel Islands. MRes Thesis, University of Kent.

Royle, J.A. (2004). N-mixture models for estimating population size from spatially replicated counts. Biometrics 60(1), 108-115.

Saha, A., McRae, L., Dodd., C.K. Gadsden, H., Hare, K.M, Lukoschek, V. \& Böhm, M. (2018). Tracking Global Population Trends: Population Time-Series Data and a Living Planet Index for Reptiles. Journal of Herpetology 52(3), 259-268.
Scott, W. A., Pithart, D. \& Adamson, J. K. (2008). Long-term United Kingdom trends in the breeding phenology of the common frog, Rana temporaria. Journal of Herpetology 42(1), 89-96.

Sheldon, S. \& Bradley, C. (1989). Identification of individual adders (Vipera berus) by their head markings. Herpetological Journal 1(9), 392-395.

Sheldon, S. (2011). Wyre Forest Adder Census and Report 1999, and Adder Population Trends Through the 1990s - Correlated with Meteorological Data. Wyre Forest Study Group, 35-39. www.wyreforest.net/wp-content/ uploads/2011/10/Reptiles-1999-SS2000.pdf

Swan, M.J.S. \& Oldham, R. S. (1993). National common reptile survey: reptile sites. Report to English Nature No. 39, vol. 2.

Taylor, R.H.R. (1963). The distribution of amphibians and reptiles in England and Wales, Scotland and Ireland and the Channel Isles: a revised survey. British Journal of Herpetology 3, 95-115.

Viitanen, P. (1967). Hibernation and seasonal movements of the viper, Vipera berus berus (L.), in southern Finland. Annales Zoologici Fennici 4, 472-546.

Ward, R.J., Griffiths, R.A., Wilkinson, J.W. \& Cornish, N. (2017). Optimising monitoring efforts for secretive snakes: a comparison of occupancy and $\mathrm{N}$-mixture models for assessment of population status. Scientific reports 7(1), 18074.

Wilkinson, J.W. \& Arnell, A.P. (2013). NARRS Report 2007-2012: Establishing the Baseline (HWM Edition). ARC Research Report 13/01.

Accepted: 10 December 2018 